Does bilingual experience influence statistical language learning?

$18 *$ Contact information: Jose A. Aguasvivas j.aguasvivas@bcbl.eu

19 BCBL, Basque Center on Cognition, Brain and Language; Paseo Mikeletegi 69, 2nd floor; 20009

20 Donostia/San Sebastian - Spain 
Abstract

Bilingual experience may confer advantages in statistical language learning tasks. Given

24 that SL tasks can measure different aspects of foreign language learning, which aspects benefit

25 from bilingual experience is still largely unexplored. Here, we compared a Spanish monolingual

26 and two (Spanish-Basque and Spanish-English) bilingual groups across three well-established SL

27 tasks. Each task targeted a different aspect of foreign language learning as a proxy-i.e., word

28 segmentation, morphological rule generalization, and word-referent learning. In Experiment 1,

29 we manipulated sub-lexical phonotactic patterns to vary the difficulty of three SL tasks, and the

30 results showed no differences between the groups in word segmentation. In Experiment 2, we

31 included non-adjacent dependencies to target affixal morphology rule learning, and again there

32 were no differences between the groups. Finally, Experiment 3 addressed word learning using a

33 more challenging audio-visual SL task combining exclusive and multiple word-referent

34 mappings. We observed a bilingual experience effect only for the exclusive mappings but not for

35 the multiple mappings. These results suggest that bilingual experience might mainly exert

36 influences on statistical language learning at the lexical level. We discuss these findings by

37 contextualizing SL as a cognitive mechanism, an experimental task, and a proxy for foreign

38 language learning, highlighting the strengths and limitations in detecting bilingual experience

39 effects.

$40 \quad$ Keywords: statistical learning, bilingualism, language learning 


\section{Does bilingual experience influence statistical language learning?}

The human brain is remarkably susceptible to structure in the environment. Both infants

44 and adults can quickly and implicitly learn from patterns presented repetitively, a mechanism

45 broadly known as statistical learning (Saffran, 2003). Statistical learning occurs regardless of the

46 input type (be it auditory, visual, or tactile), hinting towards a domain-general mechanism to

47 track and learn from regularities in the environment (Kirkham et al., 2002). The field of language

48 acquisition has taken a particular interest in statistical language learning (SL) as one of the

49 primary mechanisms humans employ to implicitly learn languages (Perruchet \& Pacton, 2006;

50 Romberg \& Saffran, 2010). However, the extent to which prior linguistic experiences affect SL

51 remains largely unexplored. Here, we examined whether one of such linguistic experiences-

52 namely, bilingualism - influences performance in SL tasks. In particular, we compared

53 monolingual and bilingual adults in three well-established SL tasks that targeted different aspects

54 of foreign language learning.

55 The idea of SL stems from observing the natural world. For example, with sufficient

56 exposure to the words in the phrase baby monkey, infants and adults learn that the syllables

57 within the words (i.e., $b a-b y$ ) predict each other more reliably than the syllables at the boundary

58 between the words (i.e., by mo) (Erickson \& Thiessen, 2015). As an experimental task, SL

59 consists of exposing participants to a continuous stream of artificial linguistic input and testing

60 them on plausible - those that follow the statistics of the input_-versus implausible items from

61 the stream (Siegelman et al., 2017). Ideally, the participants should implicitly discover the

62 boundaries between words solely from the statistics of the artificial language and mentally

63 represent them as separate units. Researchers have employed this paradigm to examine how

64 individuals implicitly learn different aspects of a novel language, including segmenting words 
65 from speech (Saffran, 2003; Saffran et al., 1996), acquiring sub-lexical units (Maye et al., 2002;

66 McMurray et al., 2009), discovering morphological rules (Frost \& Monaghan, 2016; Peña et al.,

67 2002), and learning word-referent pairs (Smith \& Yu, 2008; Yu \& Smith, 2007).

68 Individual linguistic experiences seem to influence performance in SL tasks. For

69 instance, studies have shown that the stress pattern of the native language can interfere with SL

70 performance in 9-month-old, but not in younger infants with less exposure to the native language

71 (Jusczyk et al., 1999; Thiessen \& Saffran, 2003). Adults also seem to struggle in SL tasks that

72 contain syllables that are not plausible in their native language (Finn \& Hudson Kam, 2008).

73 Furthermore, the SL performance of both infants and adults seems to be biased towards the word

74 order of their native language (Onnis et al., 2016; Onnis \& Thiessen, 2013). Overall, it seems

75 that the specific properties of a known language (e.g., stress patterns, word order) may facilitate

76 or interfere with SL performance. It makes sense that prior linguistic experiences predispose the

77 learning of novel linguistic material, as evidenced by cross-linguistic transfer studies (Alonso,

78 2016).

In bilingual adults - who consistently use two languages - , their practice with conflicting

80 statistics may influence their performance in SL tasks. To put this idea to the test, Wang and

81 Saffran (2014) compared two monolingual (English and Mandarin) and two bilingual (Mandarin-

82 English, and English-Spanish) groups in a challenging SL task that combined syllables and tones

83 to emulate a foreign tonal language. They found that, while the monolingual groups could not

84 perform above chance level in this task, both bilingual groups outperformed monolinguals. They

85 concluded that this "bilingual advantage" was irrespective of their experience with tonal

86 languages and emerged from enhanced phonological working memory in bilinguals. Additional

87 research has supported this idea using other challenging SL tasks, such as learning a Morse Code 
88 language with interfering statistics (Bartolotti et al., 2011), simultaneous learning of two

89 grammars (Onnis et al., 2018), and learning multiple word-referent pairs (Poepsel \& Weiss,

90 2016). However, by using challenging SL tasks, all of these studies fail to disentangle the effects

91 of SL task difficulty from those stemming from bilingual experience.

\section{The present study}

93 The literature presented so far highlights the influence of linguistic experience-

94 particularly of bilingual experience-, on SL tasks. Given that SL tasks can measure different

95 aspects of foreign language learning, several questions arise from this overview. First, if

96 bilingual experience facilitates SL in general, do bilinguals show an overall advantage regardless

97 of the specific SL task? Second, are the effects of bilingual experience modulated by the SL task

98 difficulty? Third, do these effects emerge at specific levels of implicit foreign language learning?

99 To address these questions, we compared monolingual and bilingual adults' performance across

100 three SL experiments. The experiments targeted three aspects of foreign language learning as a

101 proxy-i.e., word segmentation, morphological rule generalization, and word-referent learning-

102 using well-established tasks reported in the literature. Given the consistency in published

103 findings indicating the influence of bilingual experience on SL performance, our general

104 hypothesis was that bilinguals should outperform their monolingual peers in the three tasks.

105 Critically, two bilingual groups were included in the study to isolate the effects of

106 bilingual experience rather than specific language combinations. Spanish-English and Spanish-

107 Basque bilinguals were compared to each other and against a Spanish monolingual group. All

108 participants were from Spain, with Spanish-Basque bilinguals belonging to a different region

109 (Basque Country) than the Spanish and Spanish-English groups (Murcia). While prior studies

110 have emphasized the characteristics that differentiate Spanish and English, it is essential to 
111 briefly comment on the commonalities and differences between the Spanish and Basque

112 languages. Spanish and Basque do not share any common root, but Basque possesses many

113 Spanish loan words due to their geographic proximity within Spain. Some language-specific

114 bigrams differentiate Basque from Spanish (e.g., "tx", "tz"). However, the two languages are

115 phonologically similar. The Basque language also possesses a predominantly subject-object-verb

116 (SOV) word order, which involves a postpositional and agglutinative morphology-i.e.,

117 morphemes and determiners are appended to the end of word roots (e.g., eskolan - school the in).

118 These properties differentiate Basque from Spanish, English, and many other Indo-European

119 languages (Bengtson, 2011).

\section{Experiment 1}

Experiment 1 tested the word segmentation performance of monolinguals and bilinguals

123 adults in three SL streams that differed in their sub-lexical phonotactic patterns. Phonotactics

124 define the allowed combinations of phonemes in a given language. Studies have shown that

125 violating the phonotactic patterns of the native language can hinder the word learning

126 performance of infants and adults (Estes et al., 2016; Finn \& Hudson Kam, 2008). For instance,

127 exposing English-speaking adults to an SL stream containing syllables that do not exist in

128 English (e.g., /tfobu/) leads to lower performance than stimuli that only contain plausible English

129 syllables (Finn \& Hudson Kam, 2008). Sublexical phonotactics - defined here as the plausibility

130 of constituent syllables - provide an excellent way of adjusting the difficulty of SL tasks.

131 Bilinguals can potentially experience distinct phonotactics in both languages, affecting

132 their word-learning performance (Kaushanskaya \& Marian, 2009). Consequently, we expected

133 both bilingual groups to show an advantage compared to monolinguals in this task. Additionally, 
134 a specific question for this experiment was whether task difficulty could modulate this

135 advantage. Therefore, participants in this experiment listened to three SL streams. The first one

136 was constructed using simple consonant-vowel syllables, whereas the other two contained

137 consonant clusters that were either legal or illegal.

138 Methods

139 Anonymized participant information, experimental data, materials, and annotated 140 analyses scripts for all experiments are available at https://osf.io/86sr4/.

$141 \quad$ Participants

142 Forty Spanish monolinguals $\left(\mathrm{M}_{\mathrm{age}}=21.8, \mathrm{SD}=2.6 ; 37\right.$ females $)$, forty Spanish-Basque

143 bilinguals $\left(\mathrm{M}_{\mathrm{age}}=21.2, \mathrm{SD}=1.9 ; 35\right.$ females $)$, and thirty-seven Spanish-English bilinguals $\left(\mathrm{M}_{\text {age }}\right.$

$144=20.9, \mathrm{SD}=2.3 ; 33$ females) participated in this experiment. All participants reported normal or 145 corrected-to-normal vision and no history of hearing or other neurological disorders. The study 146 protocol was approved by the Ethics Committee of the Basque Center for Cognition, Brain and 147 Language (BCBL) and carried out following the Code of Ethics of the World Medical 148 Association (Declaration of Helsinki) for experiments involving humans. Before their inclusion 149 in the study, all subjects provided written informed consent. They received monetary 150 compensation for their participation.

151 Before participating in the study, they completed a language background questionnaire, a 152 picture-naming task, and a matrix reasoning task from the Kaufman Brief Intelligence Test 153 (Kaufman \& Kaufman, 2014) to measure non-verbal IQ. The linguistic questionnaire included 154 information about the age of acquisition, total exposure to each language, self-rated proficiency 155 scales for each known language, and demographic questions such as age and gender. The picture 156 naming task consisted of sixty-five images from the Basque, English, and Spanish Test (BEST) 
157 (de Bruin et al., 2017). Participants in the monolingual group named the images in Spanish and

158 English, while the bilingual groups named them in both Spanish and their L2 (English or

159 Basque). Additionally, participants in the bilingual groups completed the LexTALE test in

160 Spanish and their respective L2 (Lemhöfer \& Broersma, 2012). The combination of these tests

161 provided information on receptive and productive L2 vocabulary in the bilingual groups.

162 We compared the three groups using several ANOVAs with Helmert contrasts. In other

163 words, we contrasted the Spanish monolingual group against the two bilingual groups in the first

164 level and both bilingual groups against each other in the second level. A complete table with

165 demographic information and statistical comparisons can be found in Appendix A. The results

166 suggested that the three groups did not differ in their age $\left(p=0.204, \mathrm{BF}_{10}=0.303\right)$, non-verbal

167 IQ $\left(p=0.619, \mathrm{BF}_{10}=0.303\right)$, self-reported Spanish proficiency $\left(p=0.419, \mathrm{BF}_{10}=0.166\right)$ and

168 Spanish picture naming $\left(p=0.245, \mathrm{BF}_{10}=0.260\right)$. As expected, the monolingual and bilingual

169 groups differed in their exposure to Spanish, L2 age of acquisition, L2 exposure, L2 self-rated

170 proficiency, and BEST picture naming in L2 (all $p<0.001$ ). Crucially, the two bilingual groups

171 did not differ in their L1 and L2 exposure, age of acquisition, self-rated proficiency, BEST

172 picture naming, and LexTALE scores (all $p>0.05)$.

$173 \quad$ Materials

174 We constructed three auditory SL streams with eight trisyllabic words per stream. The 175 syllables did not repeat within the streams, maintaining the transitional probabilities within the

176 words at a constant value of 1 . The eight words for each condition were randomly concatenated 177 into continuous speech streams, with the constraint that there was no immediate word repetition 178 and that each word appeared 80 times per stream. 
The first of these streams, the simple condition, was only composed of consonant-vowel

180 (CV) syllables and represented a version of the original statistical learning experiment (Saffran et

181 al., 1996). An example of a word in this stream would be /motufi/. In the second and third

182 conditions - or complex conditions_-, each word contained one consonant cluster (CCV)

183 syllable, either at the beginning or at the end of a word. The difference between these conditions

184 was the sub-lexical phonotactic patterns that defined the syllables. In the complex legal

185 condition, the consonant cluster in the syllable (i.e., /fre/, /bla/, /fle/, /gli/, /gra/, /pre/, /pli/, /tre/)

186 were plausible at the beginning or end of a word without interfering with its segmentation (e.g.,

187 /betafre/). However, in the complex illegal condition, participants would hear words with

188 consonant clusters that generally mark the boundary between syllables (i.e., /rnu/, /gma/, /gi/,

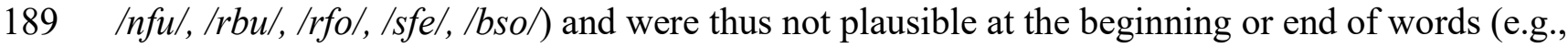

190 /tenobso/). These consonant clusters were selected because they provided interfering cues-i.e.,

191 syllable boundaries — when inserted in an uninterrupted speech.

192 We synthesized the streams and each separate word using the MBROLA software (Dutoit

193 et al., 1996), using the it3 voice, a constant pitch of $82.63 \mathrm{~Hz}$, and a duration of $200 \mathrm{~ms}$ per

194 syllable. No pauses were inserted between the words in any of the auditory streams. These

195 constraints ensured minimal interference of segmentation cues due to varying pitch, amplitudes,

196 or co-articulation of the syllables. Finally, following previous research, we introduced an

197 amplitude ramp for the first and final 5 seconds of the stream (Onnis et al., 2016). It produced a

198 fade-in and fade-out effect, giving the impression of an unbounded speech stream. The duration

199 of each SL stream was around 7 minutes.

\section{$200 \quad$ Procedure}


Participants completed a familiarization phase followed by a 2-alternatives forced-choice

202 (2AFC) test. They were asked to wear headphones and sit in a quiet room for the entire

203 experiment. During the familiarization task, they listened to the artificial SL streams. As in prior

204 studies, they were instructed to pay close attention to the made-up languages because later, they

205 would answer questions about them (Saffran et al., 1999; Wang \& Saffran, 2014). Each

206 condition's order of presentation was counterbalanced for each participant. The $2 \mathrm{AFC}$ test

207 immediately followed the familiarization phase in each condition. During this test, participants

208 heard two words (one target and one foil) separated by $500 \mathrm{~ms}$ of silence and decided which of

209 the words belonged to the previously heard language. The trials and the order of presentation of

210 target and foil words were fully randomized. They responded using the keyboard (f and $\mathrm{j}$ keys) to

211 indicate which word they thought was more similar to the previously heard language. The

212 computer automatically recorded their accuracy during the experiment.

213 We included two types of foils during the test phase, synthesized in the same manner as

214 the original words. The first type was non-words, created by inverting the syllables of the words

215 in the stream. The second type of foils was part-words. We created these foils by combining the

216 ending of one word and the beginning of another, maintaining the correspondence between

217 syllables. For instance, at test, a word in the simple condition (/bukoni/) would be paired both

218 with a non-word (/nikobu/) and with a part-word (/konito/) in separate trials. The inclusion of two

219 types of foils allowed us to double the number of trials per condition to sixteen. With three

220 familiarization and three test phases, the entire experiment lasted around 30 minutes. Participants

221 were encouraged to take a small break between each condition. The tasks for this and the rest of

222 the experiments were programmed in Psychopy (Peirce et al., 2019).

\section{Data analysis}


We analyzed participant's accuracy in the three SL streams using a generalized linear 242 factor. mixed model (GLMM). We assumed a binomial distribution and a logit link for the accuracy scores, with the Condition, Group, and their interaction as fixed effects of interest. The Condition factor was Helmert coded to contrast the simple against the complex conditions first and then contrast the two complex conditions against each other. Similarly, the Group factor was reverse Helmert coded according to our hypothesis. We first contrasted the two bilingual groups, expecting no differences between them. Then we contrasted both bilingual groups against the monolingual group. These factors were dummy-coded for each different contrast. Crucially, the contrasts were planned and coded explicitly according to our hypotheses. We decided to adopt a confirmatory approach — rather than an exploratory omnibus approach used in previous research - to evaluate the effects of bilingual experience in all experiments more robustly through a priori planned contrasts. This approach makes the GLMM analysis directly interpretable without the need for corrected post-hoc tests (Schad et al., 2020). The GLMM model was fitted using the glmer function from the "lme4" package in R (Bates et al., 2015). Following standard practice, we tried to fit the maximal random-effects structure and reduced it to achieve convergence by eliminating the correlations between random slopes or the random slopes themselves (Barr et al., 2013). The final model achieved convergence using the byparticipant and by-item intercepts with by-participant uncorrelated slopes for the Condition

Finally, we report both the frequentist and Bayesian versions of the tests where possible. We opted to report the results from the Bayesian framework because these provide robust tests of the differences between the groups while simultaneously testing for the null hypothesis (van Doorn et al., 2020). Exact p-values are reported up to the 0.001 level for frequentist tests. For 
247 Bayesian tests, we report the exact Bayes Factor $\left(\mathrm{BF}_{10}\right)$ from 0.001 to $100 . \mathrm{BF}_{10}$ values below 1

248 indicate more support for the null hypothesis, and values above 1 support the alternative

249 hypothesis. We used uninformative priors for all Bayesian analyses. Paired-, one-sample and

250 independent-samples frequentist and Bayesian tests were conducted in JASP (Love et al., 2019).

251 However, to obtain an approximate $\mathrm{BF}_{10}$ from the GLMM model, we contrasted nested models,

252 including each fixed factor and interaction step-wise, against an intercept-only model with the

253 same random-effects structure (Wagenmakers, 2007). In this case, the $\mathrm{BF}_{10}$ was calculated using

254 the Bayesian Information Criterion (BIC) of the intercept-only $\left(m_{0}\right)$ and each fixed-effect nested

255 models $\left(m_{1}\right)$ with the following formula:

$$
B F_{10}=\exp \left(\frac{B I C\left(m_{0}\right)-B I C\left(m_{1}\right)}{2}\right)
$$

\section{Results and Discussion}

We first tested whether the type of foil (i.e., non-words, part-words) influenced the SL

259 tasks' results. Wilcoxon paired-samples tests over the aggregated accuracy suggested no

260 differences between the non-word and part-word trials in the simple $\left(p=0.764, \mathrm{BF}_{10}=0.106\right)$,

261 the complex legal $\left(p=0.567, \mathrm{BF}_{10}=0.112\right)$, or the complex illegal $\left(p=0.992, \mathrm{BF}_{10}=0.104\right)$

262 conditions. Thus, we did not include a type of foil factor in the GLMM analysis. As a second

263 step, we tested whether participants' average accuracy score was above chance level (50\%) in

264 the three conditions using Wilcoxon one-sample tests. Participants in the Spanish monolingual

265 group performed above chance level in the simple $\left(\mathrm{M}_{\mathrm{acc}}=68.4, \mathrm{SD}=12.1, p<0.001, \mathrm{BF}_{10}>\right.$

266 100), complex legal $\left(\mathrm{M}_{\mathrm{acc}}=58.3, \mathrm{SD}=14.4, p<0.001, \mathrm{BF}_{10}>100\right)$, and complex illegal $\left(\mathrm{M}_{\mathrm{acc}}=\right.$

$\left.26765.0, \mathrm{SD}=12.8, p<0.001, \mathrm{BF}_{10}>100\right)$ conditions. Participants in the Spanish-English bilingual

268 group also performed above chance level in the simple $\left(\mathrm{M}_{\mathrm{acc}}=62.5, \mathrm{SD}=14.8, p<0.001, \mathrm{BF}_{10}\right.$

$269>100)$, complex legal $\left(\mathrm{M}_{\mathrm{acc}}=65.5, \mathrm{SD}=11.4, p<0.001, \mathrm{BF}_{10}>100\right)$, and complex illegal $\left(\mathrm{M}_{\mathrm{acc}}\right.$ 
$\left.270=60.1, \mathrm{SD}=13.8, p<0.001, \mathrm{BF}_{10}>100\right)$ conditions. Similarly, participants in the Spanish-

271 Basque bilingual group performed above chance level in the simple $\left(\mathrm{M}_{\mathrm{acc}}=67.3, \mathrm{SD}=17.7, p<\right.$

$\left.2720.001, \mathrm{BF}_{10}>100\right)$, legal $\left(\mathrm{M}_{\mathrm{acc}}=61.9, \mathrm{SD}=11.9, p<0.001, \mathrm{BF}_{10}>100\right)$, and illegal $\left(\mathrm{M}_{\mathrm{acc}}=\right.$

$\left.27360.6, \mathrm{SD}=13.1, p<0.001, \mathrm{BF}_{10}>100\right)$ conditions. Figure $1 \mathrm{~A}$ presents the accuracy results by

274 group and condition. Notably, participants' average performance on each task (around 60-70\%)

275 was in line with previous statistical learning studies reporting an average accuracy in the range of

$276 \quad 55-70 \%$ (Erickson \& Thiessen, 2015; Saffran, 2003).

277 


\section{Figure 1}

Average accuracy by condition and group in Experiment 1

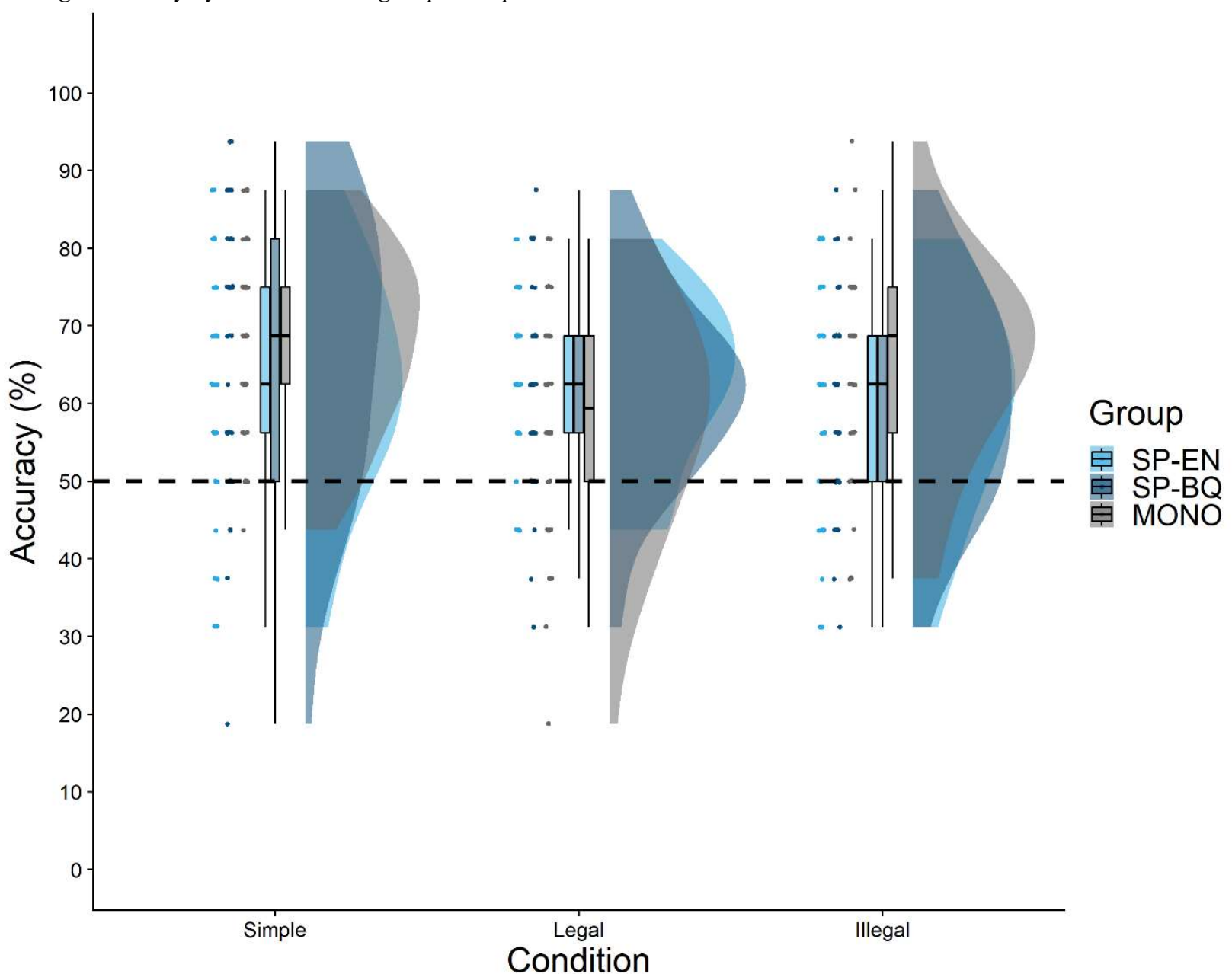

Note. Raincloud plots of accuracy scores. The center of the boxplot indicates the median, and the limits of the box define the interquartile range (IQR $=$ middle $50 \%$ of the data) for each group. Dots reflect individual participant scores. The violin plots reflect the probability density of the scores. SP-EN = Spanish-English bilinguals; SP-BQ = Spanish-Basque bilinguals; $\mathrm{MONO}=$ Spanish monolinguals.

The GLMM analysis (shown in Table 1) indicated a significant difference between the

287 simple and complex conditions $\left(p=0.001, \mathrm{BF}_{10}=2.816\right)$, but no significant differences between

288 the two complex conditions $\left(p=0.508, \mathrm{BF}_{10}=0.017\right)$. Specifically, participants performed better

289 in the simple than the complex conditions, without any differences in performance between the

290 legal and illegal complex conditions. As expected, there were no differences between Spanish-

291 Basque and Spanish-English bilingual groups $\left(p=0.425, \mathrm{BF}_{10}=0.018\right)$. However, the 
293 A significant two-way interaction indicated a difference between monolinguals and bilinguals in

294 the complex conditions $\left(p=0.010, \mathrm{BF}_{10}=0.329\right)$. This interaction seemed to be driven by the

295 monolingual group having a lower score in the complex legal compared to the complex illegal

296 conditions and is not indicative of a bilingual experience effect since both bilingual groups had

297 comparable performance in the two complex conditions.

Table 1

Accuracy GLMM results of Experiment 1

\begin{tabular}{|c|c|c|c|c|c|c|}
\hline \multicolumn{2}{|c|}{ Fixed Effects } & Estimate & SE & $\mathbf{z}$ & $p$ & $\mathbf{B F}_{10}$ \\
\hline \multicolumn{2}{|l|}{ (Intercept) } & 0.551 & 0.070 & 7.909 & $<0.001$ & - \\
\hline \multicolumn{2}{|c|}{ Simple-Complex } & 0.071 & 0.022 & 3.288 & 0.001 & 2.816 \\
\hline \multicolumn{2}{|l|}{ Legal-Illegal } & -0.023 & 0.034 & -0.662 & 0.508 & 0.017 \\
\hline \multicolumn{2}{|l|}{ SPBQ-SPEN } & 0.036 & 0.045 & 0.797 & 0.425 & 0.018 \\
\hline \multicolumn{2}{|l|}{ MONO-BIL } & 0.022 & 0.026 & 0.845 & 0.398 & 0.018 \\
\hline \multicolumn{2}{|c|}{ Simple-Complex x SPBQ-SPEN } & 0.038 & 0.027 & 1.411 & 0.158 & 0.036 \\
\hline \multicolumn{2}{|c|}{ Simple-Complex x MONO-BIL } & 0.016 & 0.015 & 1.029 & 0.304 & 0.022 \\
\hline \multicolumn{2}{|c|}{ Legal-Illegal x SPBQ-SPEN } & -0.012 & 0.042 & -0.287 & 0.774 & 0.014 \\
\hline \multicolumn{2}{|c|}{ Legal-Illegal x MONO-BIL } & -0.062 & 0.024 & -2.566 & 0.010 & 0.329 \\
\hline Random Effects & Group & Variance & $S D$ & & & \\
\hline Item & (Intercept) & 0.056 & 0.237 & & & \\
\hline \multirow[t]{3}{*}{ Participant } & (Intercept) & 0.063 & 0.250 & & & \\
\hline & Simple-Complex & 0.008 & 0.088 & & & \\
\hline & Legal-Illegal & 0.002 & 0.040 & & & \\
\hline
\end{tabular}

Note. Significant fixed effects terms are highlighted in bold. $\mathrm{SE}=$ standard error; $\mathrm{SD}=$ standard deviation; $\mathrm{SPEN}=$ Spanish-English bilinguals; SPBQ = Spanish-Basque bilinguals; MONO = Spanish monolinguals; BIL = bilinguals.

Experiment 1 revealed that more complex sub-lexical phonotactic patterns could modulate the word segmentation difficulty in SL tasks. All three groups, on average, performed the tasks above chance level. However, their performance was lower in the complex than the simple condition, with no differences between the two complex conditions. More importantly, the SL performance of monolingual and bilingual participants did not significantly differ in any of the conditions. A possible explanation for these findings is that we constructed the stimuli using syllables that naturally occur in Spanish, English, or Basque. Even if the complex illegal condition included syllables that could have interfered with the statistics in the speech stream, 
311 these syllables also appear within words in all three languages. Therefore, our task may not have

312 been challenging enough to elucidate the differences shown in previous studies (Bartolotti et al.,

313 2011; Wang \& Saffran, 2014). In other words, the bilingual experience might still come into play

314 when the task is sufficiently challenging - i.e., by using tones or completely illegal phonotactic

315 patterns - or produces interference with previously learned materials. With this in mind, in

316 Experiment 2, we increased the difficulty of the task by testing monolinguals and bilinguals

317 when extracting rules rather than specific words from an SL task designed as a proxy for 318 morphological rule learning.

\section{Experiment 2}

In the previous experiment, learning the words largely depended on the transitional

322 probabilities of adjacent syllables. Specifically, the patterns presented in these SL streams could

323 not be generalized outside of their respective artificial speech streams. While language learning

324 requires identifying and learning the patterns within and across different words, it also

325 encompasses learning the morphological rules that bind them together. Learning these rules

326 allows generalization to instances where the words vary due to their dependency on other

327 constituents (Endress \& Bonatti, 2016; Peña et al., 2002). The perfect example is affixal

328 morphology, where structures append to the beginning (prefix) or ending (suffix) of a word to

329 change its class or meaning (e.g., untouchable, unbreakable). These rules can span multiple

330 elements, and in the SL literature, they are commonly known as non-adjacent dependencies

331 (Misyak \& Christiansen, 2007). Some authors posit similar SL mechanisms to those employed in

332 word segmentation underly learning these non-adjacent dependencies (Frost \& Monaghan, 2016;

333 Misyak \& Christiansen, 2007). However, to our knowledge, no prior study has addressed 
334 whether bilingual experience can affect the implicit learning and generalization of non-adjacent 335 dependencies.

336 In Experiment 2, we explored the capacity of monolinguals and bilinguals to learn non-

337 adjacent dependencies from an SL stream and generalize them to novel items. Having observed

338 no differences arising from bilingual experience in the first experiment, the purpose behind this

339 manipulation was to test whether the groups would differ in another aspect of language learning

340 by using this task as a proxy for morphological rule generalization. Since this task tested

341 participants' generalization ability and was more challenging than the previous two tasks, we

342 adhered to our original hypothesis and expected to find differences between the groups.

\section{Methods}

\section{Participants}

345 Forty Spanish monolinguals $\left(\mathrm{M}_{\mathrm{age}}=21.7, \mathrm{SD}=2.4 ; 35\right.$ females $)$, forty Spanish-Basque

346 bilinguals $\left(\mathrm{M}_{\mathrm{age}}=21.8, \mathrm{SD}=2.2 ; 32\right.$ females $)$, and forty Spanish-English bilinguals $\left(\mathrm{M}_{\mathrm{age}}=\right.$

$34721.0, \mathrm{SD}=2.4 ; 36$ females) participated in Experiment 2. Participants had a similar profile as

348 those in Experiment 1. Appendix B shows the demographic information and statistical contrasts

349 between groups.

$350 \quad$ Materials

351 We concatenated nine words with an AXC form, where A and $\mathrm{C}$ established a frame with 352 constant syllables, and X was a filler syllable that could vary. Following previous work (Frost \& 353 Monaghan, 2016), we used plosive syllables for the A_C frames (/ke/, /po/, /bi/, /ga/, /du/, /ti/) 354 and continuants for the $\mathrm{X}$ fill syllables $(/ \mathrm{mu} /, / \mathrm{fe} /, / \mathrm{li} /)$. The frame syllables were randomized to 355 create five counterbalanced versions of the SL stream. There were three different frames 356 combined with the three fill syllables, creating nine words for each version. We manually 
357 verified that none of the streams contained words or parts of words that existed in Spanish,

358 English, or Basque. There were 100 repetitions of each word in every version of the stream. We

359 synthetized the streams in the same manner as in Experiment 2, ensuring this time that there

360 were no immediate repetitions of words with the same frame. The overall duration of the speech

361 streams was about 12 minutes.

362 Procedure

363 The procedure was identical to the previous experiment except for the test trials. For the

364 2AFC test, we used three continuant syllables as generalization fills (/se/, /ya/, /ni/) and inserted

365 them in the original frames to produce nine AYC generalization words. We tested participants on

366 their ability to distinguish these words from three types of foils. The first two types were part-

367 words created by concatenating the ending of the target word and the beginning of a different

368 word from the stream. For example, a target word (/kenipo/) would be paired with a part-word

369 starting at the fill syllable (/nipoti/) or ending with the fill syllable (/gakeni/). The last type of

370 trials were non-words, created by inverting the word's syllables (e.g., /ponike/). Due to the

371 randomized nature of the different versions, a target word in one of the lists could potentially be

372 a non-word foil in any other version. The inclusion of three types of tests increased the number

373 of trials to 27 , representing a substantial increase from the previous experiment.

\section{$374 \quad$ Data analysis}

375 The data analysis closely followed Experiment 1, the only difference being that there

376 were no conditions.

\section{Results and Discussion}

As a first step, we tested whether the list or the type of foil influenced average

379 performance in the task. An ANOVA suggested similar performance regardless of the stream's 
380 version $\left(p=0.069 ; \mathrm{BF}_{10}=0.535\right)$ and the type of foil $\left(p=0.527, \mathrm{BF}_{10}=0.052\right)$, and no

381 interaction between the two $\left(p=0.217, \mathrm{BF}_{10}=0.009\right)$. Furthermore, Wilcoxon one-sample tests

382 against chance-level indicated that participants in the monolingual $\left(\mathrm{M}_{\mathrm{acc}}=59.1, \mathrm{SD}=17.5, p=\right.$

$\left.3830.011, \mathrm{BF}_{10}>100\right)$, Spanish-English $\left(\mathrm{M}_{\mathrm{acc}}=57.3, \mathrm{SD}=13.4, p=0.005, \mathrm{BF}_{10}>100\right)$, Spanish-

384 Basque $\left(\mathrm{M}_{\mathrm{acc}}=60.1, \mathrm{SD}=15.1, \mathrm{p}<0.001, \mathrm{BF}_{10}>100\right)$ groups performed above the $50 \%$

385 chance-level in the task. Figure 2 depicts the results by group. In this case, the GLMM revealed 386 no differences between the two bilingual groups (Estimate $=0.066, \mathrm{SE}=0.077, z=0.858, p=$

$\left.3870.391, \mathrm{BF}_{10}=0.025\right)$, or between the monolingual and bilingual groups (Estimate $=0.009, \mathrm{SE}=$ $\left.3880.044, z=0.216, p=0.829, \mathrm{BF}_{10}=0.018\right)$. 


\section{Figure 2}

391

Average accuracy by Group in Experiment 2

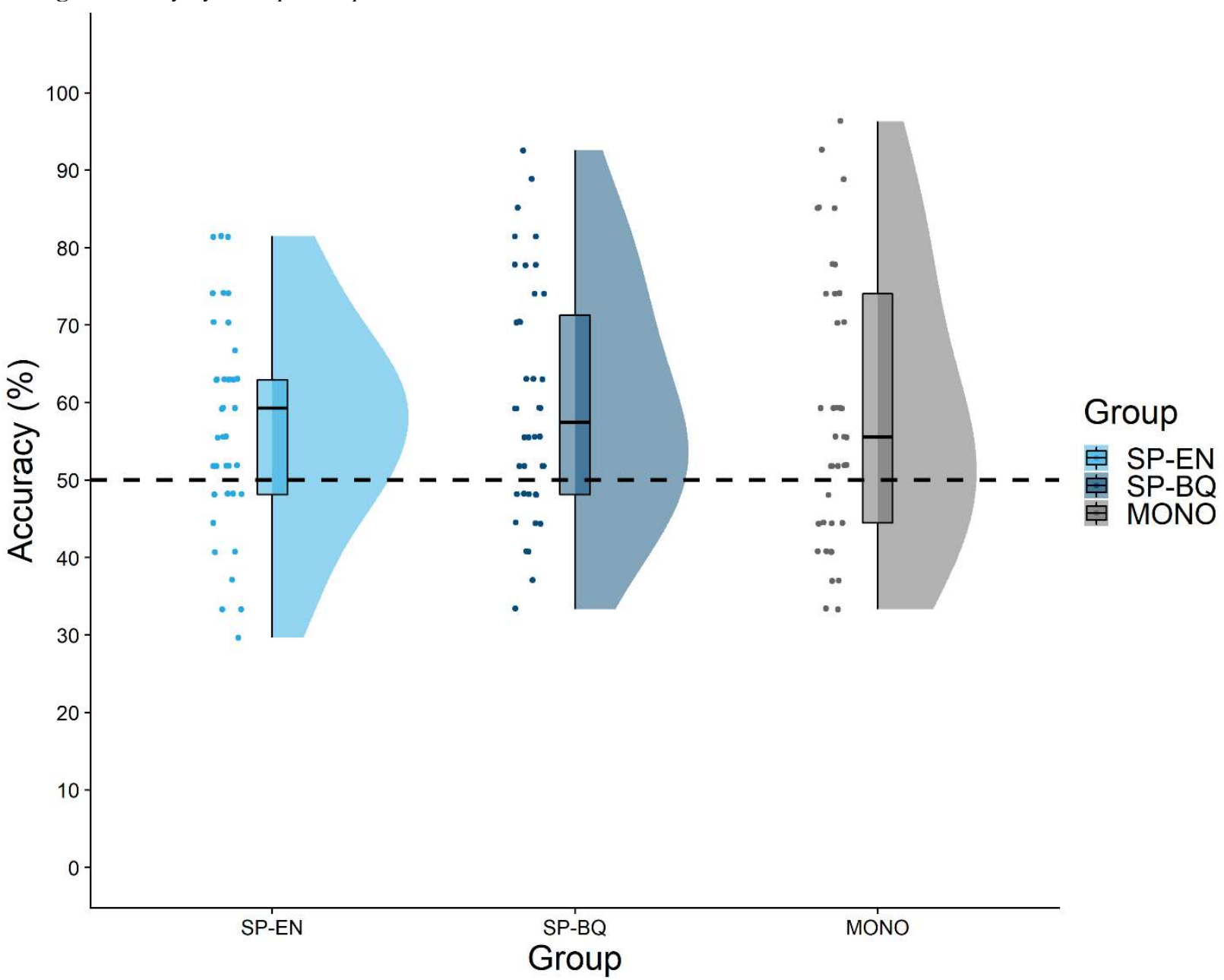

Note. Raincloud plots of accuracy scores. The center of the boxplot indicates the median, and the limits of the box define the interquartile range (IQR $=$ middle $50 \%$ of the data) for each group. Dots reflect individual participant scores. The violin plots reflect the probability density of the scores. SP-EN = Spanish-English bilinguals; SP-BQ = Spanish-Basque bilinguals; $\mathrm{MONO}=$ Spanish monolinguals.

Overall, Experiment 2 extended the findings from the previous experiment by testing the generalization of non-adjacent dependencies learned during an SL task as a proxy for morphological rule learning. In line with previous studies, participants could generalize the

401 frames extracted from the artificial language above chance-level, hinting towards similar implicit 402 mechanisms for word and rule learning (Endress \& Bonatti, 2016; Frost \& Monaghan, 2016).

403 However, the focal point of Experiment 2 was to compare the performance of bilinguals and 404 monolinguals in generalizing information from the SL task. The main challenge of this 
experiment was that participants needed to extract the non-adjacent frames from the stream-

406

407

408 effects.

409

410

411

412 knowledge of Spanish. In other words, the three languages may not differ sufficiently in their

413 affixal morphology, such as to affect the non-adjacent dependencies generalization measured by

414 this SL task. Notably, we did not target any specific differences that long-term speakers of two

415 languages might exploit for a learning advantage. Thus, even if the task was more challenging

416 and addressed a different aspect of language compared to the previous experiment, we still did

417 not observe effects stemming from the bilingual experience. A possibility raised by prior work is

418 that bilingual experience might mainly influence learning at the lexical level (Kaushanskaya \&

419 Marian, 2009; Poepsel \& Weiss, 2016). Hence, in Experiment 3, we presented participants with

420 an audio-visual SL task targeting word-referent learning.

426 other SL tasks because participants learn the names for different visual referents by aggregating

427 information through multiple contexts. In other words, they implicitly "discover" each object's 
428 name by seeing it in conjunction with other distinct referents. This task was designed to mimic

429 the crowded visual world where infants initially learn words (Yu \& Smith, 2007). It seems to

430 target a crucial implicit mechanism for learning words in native and foreign languages (Benitez

431 et al., 2016; Smith \& Yu, 2008).

432 Perhaps one of the defining characteristics of bilingual experience is learning to map one

433 referent to two potentially different words (e.g., perro-dog/txakur), but this phenomenon is not

434 unique to bilingualism and happens within languages. For instance, there are concepts with

435 multiple names or synonyms (e.g., paper - sheet) and words with multiple referents or homonyms

436 (e.g., bat as the animal or the object). Bilinguals have the added difficulty of learning these two

437 types of mappings within two languages and across languages, potentially influencing their

438 ability to learn them. Prior work has shown that bilinguals can outperform monolinguals in a

439 CSSL task when learning homonyms (i.e., one word to two referents), but not when learning

440 exclusive one-to-one mappings (Poepsel \& Weiss, 2016). Other authors only report differences

441 between bilinguals and monolinguals in the one-to-one mappings (Escudero et al., 2016).

442 Finally, some have only found minimal differences between bilinguals and monolinguals

443 learning one-to-one and synonym (i.e., two words to one referent) mappings (Benitez et al., 444 2016).

445 To address these discrepant findings, in Experiment 3, we compared monolinguals and 446 bilinguals in a CSSL task that entailed learning one-to-one, two-to-one (synonyms), and one-to447 two (homonyms) word-referent pairs. If bilingual experience facilitates overall word learning, 448 participants in the bilingual groups should outperform those in the monolingual group in the one449 to-one mappings. However, if bilingual experience only potentiates learning multiple word- 
450 referent mappings, they should perform better than monolinguals in the more challenging

451 multiple mapping conditions.

452 Methods

453 Participants

454 Forty Spanish monolinguals $\left(\mathrm{M}_{\mathrm{age}}=21.7, \mathrm{SD}=2.5 ; 37\right.$ females $)$, forty Spanish-Basque

455 bilinguals $\left(\mathrm{M}_{\mathrm{age}}=21.9, \mathrm{SD}=1.9 ; 31\right.$ females $)$, and thirty-seven Spanish-English bilinguals $\left(\mathrm{M}_{\mathrm{age}}\right.$

$456=21.0, \mathrm{SD}=2.4 ; 32$ females) participated in Experiment 3. Participants had a similar profile as

457 those in Experiment 1. Appendix C shows the demographic information and statistical contrasts

458 between groups.

459 Materials

460 We created 30 words by randomly concatenating letters from a pool of consonants $(\mathrm{t}, 1, \mathrm{k}$,

$461 \mathrm{~m}, \mathrm{n}, \mathrm{b}, \mathrm{s}, \mathrm{p}, \mathrm{g}, \mathrm{d})$ and vowels $(\mathrm{a}, \mathrm{i}, \mathrm{u}, \mathrm{e}, \mathrm{o})$ in an alternating manner. The words had either a

$462 \operatorname{CVCVCV}$ (e.g., /ninugo/) or a VCVCV (e.g., /udili/) form and were synthesized using the Mac

463 OS X system's Text-to-Speech software with the Spanish female voice "Monica". Since these

464 words were presented in isolation, we did not enforce as much control (e.g., constant pitch,

465 vowel duration) as in the previous three experiments, thus creating more natural-sounding

466 stimuli. We eliminated words that existed or sounded like actual Spanish, English, or Basque

467 words from an initial list. The final 30 words were paired with 30 color depictions of non-

468 existing objects manually selected from the NOUN database (Horst \& Hout, 2016) based on their

469 color saliency and visual complexity scores.

470 The critical manipulation in this task involved creating the different word-referent

471 mappings. As shown in Figure 3, twelve words were randomly paired with twelve objects to

472 form the exclusive (one-to-one) mappings. For the homonym (one-to-two) mappings, we 
473 randomly paired six words and twelve objects, producing words with two distinct referents.

474 Conversely, for the synonym (two-to-one) mappings, twelve words were randomly paired with

475 six objects, such that each of these objects had two different names. We manually verified that

476 the words and objects in the multiple mapping conditions were not very similar to avoid

477 artificially increasing task difficulty.

Figure 3

Word-referent mappings in Experiment 3

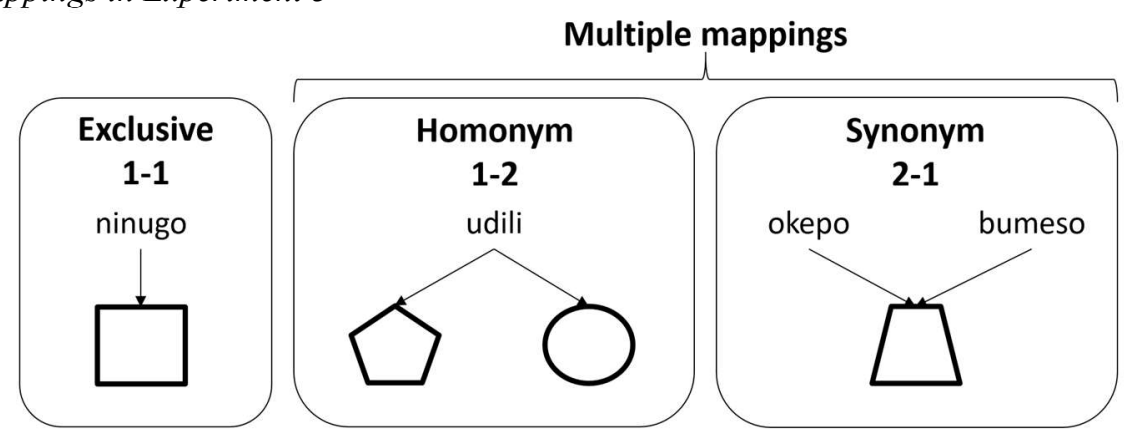

480

481

482

483

484

485

486

487

488

489

490

491

492

493

494

495

496

497

Note. The leftmost panel depicts an example of the Exclusive one-to-one mappings, where one word was consistently presented with one visual referent. The middle panel illustrates an example of a Homonym one-to-two mapping, where one word was paired with two distinct visual referents. The rightmost panel shows an example of a Synonym two-to-one mapping, where two words referred to the same visual referent. The depicted words are actual examples from the experiment, but we replaced the objects from the NOUN dataset with simple geometric figures for illustrative purposes.

\section{Procedure}

Participants completed three blocks of alternating familiarization and test phases. As in previous experiments, they were asked to wear headphones and sit in a quiet room. In each familiarization phase, they saw thirty-two scenes composed of three objects horizontally aligned at the center of the screen over a white background. They also heard the three names for the depicted objects in each scene, starting after one second and with a one-second pause between them. Participants were instructed to pay attention to the different scenes because they would later perform a test based on them. The objects' position and the order of the audios were fully randomized in each scene so that there was no particular match between words and referents. Following previous studies (Poepsel \& Weiss, 2016), only one side of the multiple mappings was 
498 presented during the first half of the scenes. During the second half, the other side of the multiple

499 mappings was shown, simulating a change of context. This manipulation allowed us to test the

500 effects of primacy and recency on the multiple mappings. We created four counterbalanced lists

501 by varying which side of the two multiple mapping conditions was presented during the first and

502 second halves of the familiarization phase. Thus, the scenes' order of presentation was pseudo-

503 randomized for each familiarization phase so that no word or referent, including two sides of the

504 multiple mappings, would appear in contiguous scenes.

505 Following each familiarization phase, participants completed a 2AFC test. In each trial,

506 two horizontally-aligned objects (one target and one foil) were presented, and the target object's

507 name was played. The trials' order of presentation and the position of target and foil objects were

508 fully randomized. Participants were instructed to select the object they thought corresponded to

509 the heard name using the keyboard ("fo" and " $\mathrm{j}$ " keys). The one-to-one mappings were tested

510 during the three test blocks. However, during the first two experimental blocks, only one side of

511 the multiple mappings was presented, corresponding to the order of presentation during the

512 familiarization phase (Poepsel \& Weiss, 2016). The complete word-referent list was used for the

513 last block, controlling that the foil object did not correspond to the multiple mapping condition.

514 The entire experiment lasted about 45 minutes, and participants were encouraged to take small

515 breaks between each block.

516 Data analysis

517 We modeled the exclusive and multiple (synonyms/homonyms) mappings data separately

518 using GLMMs, similar to the previous two experiments. For the exclusive mappings, we treated

519 the block as a continuous linear factor and compared the reverse Helmert-coded groups and their

520 interaction with the blocks as fixed effects. In this case, the GLMM was performed over the 
521 proportion correct data to avoid inflating the estimates for the linearized block (Mirman, 2017).

522 As in the previous experiments, we contrasted the Spanish-Basque against the Spanish-English

523 group first, and then both bilingual groups against monolinguals. We included the group, the

524 block, and their interactions as fixed effects and the by-participant intercept and uncorrelated

525 block slope as random effects.

526 For the more challenging multiple mappings, since participants were tested on different

527 mapping sides during the first and second blocks, we only contrasted the groups in the final test

528 at block three. We created a factor to account for the order of presentation in the CSSL task.

529 During the familiarization phase, the mappings displayed first represented the primacy

530 mappings, and those displayed later the recency mappings. The group contrasts were the same as

531 for the exclusive mappings. We tested the order-of-presentation factor and its interactions with

532 the group as fixed effects. We included the by-participant and by-item intercepts and the by-

533 participant slope for order of presentation as random effects in the final GLMMs.

\section{Results and Discussion}

535 Before the analysis, we removed one participant from the monolingual group with chance

536 level performance in all three exclusive blocks. We first confirmed there were no differences

537 between the lists across the three blocks on the exclusive mappings, as the counterbalanced lists

538 only targeted the multiple mappings. A series of ANOVAs revealed that there were no

539 differences due to the list in the first $\left(p=0.901, \mathrm{BF}_{10}=0.058\right)$, second $\left(p=0.586, \mathrm{BF}_{10}=0.096\right)$,

540 or third block $\left(p=0.815, \mathrm{BF}_{10}=0.066\right)$. Next, we compared participants' accuracy on the

541 Exclusive mappings to chance level (50\%) in the last block. Wilcoxon one-sample tests indicated

542 that Spanish monolinguals $\left(\mathrm{M}_{\mathrm{acc}}=83.1, \mathrm{SD}=14.6, p<0.001, \mathrm{BF}_{10}>100\right)$, Spanish-English

543 bilinguals $\left(\mathrm{M}_{\mathrm{acc}}=86.9, \mathrm{SD}=14.2, p<0.001, \mathrm{BF}_{10}>100\right)$, and Spanish-Basque bilinguals $\left(\mathrm{M}_{\mathrm{acc}}\right.$ 
$\left.544=91.0, \mathrm{SD}=10.2, p<0.001, \mathrm{BF}_{10}>100\right)$ performed significantly above chance level. Figure 4A

545 depicts the accuracy for each group across the three blocks. Notably, accuracy was higher for

546 this task than in the previous experiments.

547 


\section{Figure 4}

Average accuracy by group and block in Experiment 3

A

A Exclusive



Group 皇 SP-EN

B Homonyms

Synonyms



Note. (A) Accuracy by group and block in the one-to-one (Exclusive) mappings. (B) Accuracy by group, mapping type, and order of presentation for the multiple (Homonyms and Synonyms) mappings. The center of the boxplot indicates the median, and the limits of the box define the interquartile range (IQR $=$ middle $50 \%$ of the data) for each group. Dots reflect individual participant scores. The violin plots reflect the probability density of the scores. $\mathrm{SP}-\mathrm{EN}=$ Spanish-English bilinguals; $\mathrm{SP}-\mathrm{BQ}=$ Spanish-Basque bilinguals; $\mathrm{MONO}=$ Spanish monolinguals . 
Table 3

Accuracy GLMM results of exclusive condition in Experiment 4

\begin{tabular}{|c|c|c|c|c|c|c|}
\hline Fixed Effects & & Estimate & SE & $\mathbf{z}$ & $p$ & $\mathrm{BF}_{10}$ \\
\hline (Intercept) & & 1.411 & 0.064 & 22.141 & $<0.001$ & - \\
\hline Block & & 0.938 & 0.076 & 12.371 & $<0.001$ & $>100$ \\
\hline SPBQ-SPEN & & 0.149 & 0.078 & 1.927 & 0.054 & 0.190 \\
\hline MONO-BIL & & -0.124 & 0.043 & -2.886 & 0.004 & 1.902 \\
\hline Block x SPBQ-SPEN & & 0.163 & 0.095 & 1.720 & 0.085 & 0.079 \\
\hline Block x MONO-BIL & & -0.079 & 0.051 & -1.542 & 0.123 & 0.061 \\
\hline Random Effects & Group & Variance & $S D$ & & & \\
\hline \multirow[t]{2}{*}{ Participant } & (Intercept) & 0.250 & 0.500 & & & \\
\hline & Linear & 0.059 & 0.243 & & & \\
\hline
\end{tabular}

Note. Significant fixed effects terms are highlighted in bold. $\mathrm{SE}=$ standard error; $\mathrm{SD}=$ standard deviation; $\mathrm{SPEN}=$ Spanish-English bilinguals; $\mathrm{SPBQ}=$ Spanish-Basque bilinguals; $\mathrm{MONO}=$ Spanish monolinguals; $\mathrm{BIL}=$ bilinguals .

Table 3 shows the results for the exclusive mappings' GLMM. Unsurprisingly, the analysis revealed a significant effect of the block $\left(p<0.001, \mathrm{BF}_{10}>100\right)$, indicating that participant's accuracy increased across the three blocks. The results also indicated no significant differences between the two bilingual groups $\left(p=0.054, \mathrm{BF}_{10}=0.190\right)$. However, there were significant differences between monolinguals and bilinguals $\left(p=0.004, \mathrm{BF}_{10}=1.902\right)$. The interactions between block and group did not reach statistical significance $\left(p=0.085, \mathrm{BF}_{10}=\right.$ $0.079 ; p=0.123, \mathrm{BF} 10=0.061 ;$ respectively $)$.

In the homonym condition (Figure 4B, left), Wilcoxon one-sample tests indicated that participants in the monolingual $\left(\mathrm{M}_{\mathrm{acc}}=82.3, \mathrm{SD}=14.6, p<0.001, \mathrm{BF}_{10}>100\right)$, Spanish-Basque $\left(\mathrm{M}_{\mathrm{acc}}=87.1, \mathrm{SD}=12.1, p<0.001, \mathrm{BF}_{10}>100\right)$, and Spanish-English $\left(\mathrm{M}_{\mathrm{acc}}=80.6, \mathrm{SD}=17.9, p\right.$ $\left.<0.001, \mathrm{BF}_{10}>100\right)$ groups performed above the $50 \%$ chance level on average. The GLMM results for the homonym mappings indicated a significant effect of the order of presentation (Estimate $\left.=-0.359, \mathrm{SE}=0.113, \mathrm{z}=-3.173, p=0.002, \mathrm{BF}_{10}=4.874\right)$, where performance in the recency mappings was, on average, better than in the primacy mappings. There were no differences between the two bilingual groups (Estimate $=0.236, \mathrm{SE}=0.145, \mathrm{z}=1.627, p=$ $\left.0.104, \mathrm{BF}_{10}=0.148\right)$, or between the monolingual and bilingual groups $($ Estimate $=-0.063, \mathrm{SE}=$ 
$\left.5770.082, \mathrm{z}=-0.769, p=0.442, \mathrm{BF}_{10}=0.034\right)$. There were no significant interactions between the

578 order of presentation and the group contrasts (all $\left.p>0.05, \mathrm{BF}_{10}<0.1\right)$.

579 In the synonym condition, (Figure 4B, right), Wilcoxon one-sample tests indicated that

580 participants in the monolingual $\left(\mathrm{M}_{\mathrm{acc}}=67.7, \mathrm{SD}=16.0, p<0.001, \mathrm{BF}_{10}>100\right)$, Spanish-Basque

$581 \quad\left(\mathrm{M}_{\mathrm{acc}}=73.5, \mathrm{SD}=14.2, p<0.001, \mathrm{BF}_{10}>100\right)$, and Spanish-English $\left(\mathrm{M}_{\mathrm{acc}}=66.9, \mathrm{SD}=13.5, p\right.$

$\left.582<0.001, \mathrm{BF}_{10}>100\right)$ groups performed above the $50 \%$ chance level on average. The GLMM for

583 this condition revealed an effect of order of presentation in the same direction as for the

584 homonym mappings $\left(\right.$ Estimate $\left.=-0.286, \mathrm{SE}=0.068, \mathrm{z}=-4.211, p<0.001, \mathrm{BF}_{10}>100\right)$.

585 However, in this case, the analysis revealed a marginally significant difference between Spanish-

586 Basque and Spanish-English bilinguals $\left(\right.$ Estimate $=0.174, \mathrm{SE}=0.086, \mathrm{z}=2.023, p=0.043, \mathrm{BF}_{10}$

$587=0.211)$, and no difference between monolinguals and bilinguals (Estimate $=-0.037, \mathrm{SE}=$

$\left.5880.079, \mathrm{z}=-0.752, p=0.452, \mathrm{BF}_{10}=0.037\right)$. As in the homonym condition, there were no

589 significant interactions between the order of presentation and the group contrasts (all $p>0.05$,

$\left.590 \quad \mathrm{BF}_{10}<0.1\right)$. Additionally, a Wilcoxon paired-samples test confirmed that accuracy was overall

591 higher in the homonym than in the synonym mappings $\left(p<0.001, \mathrm{BF}_{10}>100\right)$.

592 Overall, Experiment 3 indicated that participants could learn the exclusive mappings and

593 the two types of multiple mappings from the same audio-visual CSSL task. Contrary to our two

594 previous experiments, this design also allowed us to test participants' learning at three stages,

595 showing a progressive increase in their performance for the exclusive mappings. In this regard,

596 both bilingual groups outperformed the monolingual group on the exclusive one-to-one

597 mappings. Thus, our results align with prior findings suggesting bilingual experience effects for

598 these types of mappings in CSSL and other explicit word-learning tasks (Escudero et al., 2016;

599 Kaushanskaya \& Marian, 2009). However, these findings should be interpreted in the context of 
600 the more challenging multiple mappings. The task itself was more demanding than the previous

601 experiments - i.e., participants had to learn thirty novel items and words with different mappings

602 in an implicit manner. Therefore, it is unclear whether the observed bilingual experience effect is

603 constrained to the exclusive mappings or emerged due to the conflicting multiple mapping

604 conditions.

605 Despite a similar design, our results diverge from prior work reporting bilingual

606 experience effects in the homonym but not in the exclusive mappings (Poepsel \& Weiss, 2016).

607 The homonym mappings were easier to acquire for our participants than the synonym mappings,

608 and there were no differences between monolinguals and bilinguals on these multiple mapping

609 conditions. A possible reason for these findings is that pure synonyms - especially those

610 referring to concrete objects with the same meaning - are significantly rarer than homonyms in

611 most natural languages (Hurford, 2003). Moreover, it is often necessary to introduce additional

612 cues, such as speaker identity or spacing between presentations, to avoid direct competition from

613 multiple mappings during learning (Benitez et al., 2016). Out of these two factors, we only

614 spaced the multiple mappings' presentations during the learning phase, leading to the observed

615 difference between primacy and recency mappings extensively reported in associative learning

616 research (Pineño \& Miller, 2005). These results suggest exciting avenues for investigating the

617 role of other cues in how bilinguals and monolinguals learn multiple mappings, but these remain 618 beyond the scope of our study.

\section{General Discussion}

The present study examined the effects of bilingual experience across three statistical

622 language learning (SL) experiments. We opted to use three well-established SL tasks to 
623 approximate different aspects of foreign language learning as a proxy, including word

624 segmentation from continuous speech (Experiment 1), morphological rule generalization

625 (Experiment 2), and word-referent learning (Experiment 3). The inclusion of two bilingual

626 groups and different manipulations allowed us to disentangle the overall effects of bilingual

627 experience from those stemming from task difficulty or specific language pairs. Additionally, our

628 confirmatory analytical approach and sample size — of almost three times the size reported in the

629 literature - facilitated the straightforward evaluation of our central hypothesis.

630 Overall, Experiment 1 showed that, while manipulating the sub-lexical phonotactics of

631 the SL stream affected participants' performance, there were no bilingual experience effects for

632 word segmentation. In Experiment 2, participants generalized the learned non-adjacent

633 dependencies during the test phase, but again, we did not find any bilingual experience effects.

634 Lastly, in Experiment 3, we tested participants' ability to learn exclusive and multiple word-

635 referent pairs across three blocks. The results revealed that bilingual participants outperformed

636 their monolingual peers when learning the exclusive but not the multiple word-referent

637 mappings. Notably, in all experiments, regardless of the manipulation, the average performance

638 of all groups was significantly above chance and in the range reported in the literature (Erickson

$639 \&$ Thiessen, 2015; Perruchet \& Pacton, 2006; Saffran, 2003), which points to the robustness of

$640 \mathrm{SL}$ as an implicit mechanism for foreign language learning.

641 It is essential to distinguish SL as a cognitive mechanism, an experimental task, and a

642 proxy for foreign language learning. As a cognitive mechanism, other individual differences

643 could perhaps account for auditory SL performance better than the bilingual experience, such as

644 spontaneous synchronization to speech (Assaneo et al., 2019). In other words, some intrinsic

645 particularities of the cognitive system could influence SL as a cognitive mechanism. Also, 
646 bilingual experience effects may be reduced or eliminated in the absence of interfering

647 information. Experiments 1 and 2 showed no bilingual experience effects, but the differences in

648 Experiment 3 could have emerged in the context of the more difficult multiple mapping

649 conditions. Prior studies seem to support this idea, showing bilingual experience effects only

650 when participants learn from interfering SL streams (Bartolotti et al., 2011; Onnis et al., 2018).

651 However, the purpose of this study was to isolate the effects of bilingual experience from other

652 factors (e.g., task difficulty, interference, specific language properties) previously shown in the

653 literature (Bartolotti et al., 2011; Onnis et al., 2018; Wang \& Saffran, 2014). In doing so, we

654 purposefully refrained from introducing additional variables that could more likely benefit from

655 the bilingual experience. For instance, other studies have shown that bilingual experience could

656 influence non-native phonetic learning and conflict resolution (Antoniou et al., 2015; Donnelly et

657 al., 2015). These abilities could indirectly affect SL as a cognitive ability, but whether they

658 account for bilingual experience effects in SL tasks remains to be explored.

Considering SL simply as an experimental task, participants are sensitive to specific and

660 controlled manipulations of the probabilities between and within words that might target

661 properties of a specific language (Finn \& Hudson Kam, 2008; Onnis \& Thiessen, 2013).

662 Consequently, any bilingual experience effects may primarily rely on the properties of

663 participants' known languages and not the overall bilingual experience. Moreover, bilingual

664 experience effects might emerge progressively through learning, and one-shot 2AFC tests are not

665 sensitive enough to capture them. Most SL studies base their results on a single familiarization

666 and test phase, constraining their findings to what is learned upon first exposure to a foreign

667 language (Romberg \& Saffran, 2010). A common criticism with this approach is that

668 performance, as measured by a one-shot $2 \mathrm{AFC}$ test, is highly variable and noisy, partly due to 
669 the low number of trials generated from these artificial streams (Siegelman \& Frost, 2015). In

670 other words, there usually are only a handful of target and foil words presented during the test

671 phase, and using a single test introduces additional variability in participants' scores. Thus, while

672 these experimental tasks could be adequate to measure learning at the group level, they might not

673 be sensitive enough to detect individual differences (Siegelman \& Frost, 2015). Notably, we

674 partially addressed these limitations in Experiment 3 by adding three familiarization and test

675 phases for the twelve exclusive mappings and found differences between monolinguals and

676 bilinguals. However, observing any effects in other SL tasks might be unlikely due to the

677 variability in responses from one-shot 2 AFC tests. Future research could further address these

678 gaps by using continuous measures to focus on the process rather than the learning outcome.

679 Lastly, considering SL as a proxy for foreign language learning, bilingual experience

680 effects might arise mainly at the lexical level. Most of the literature supporting a bilingual

681 advantage primarily focuses on explicit vocabulary learning tasks, highlighting that bilinguals

682 might more easily establish links between new word forms (orthographic or phonological) and

683 their meanings within the mental lexicon than monolinguals (Antoniou et al., 2015;

684 Kaushanskaya \& Marian, 2009). In this regard, the present study showed no bilingual experience

685 effects using SL tasks as proxies for word segmentation and morphological levels. However,

686 there were significant differences at the lexical level in the word-referent learning task. Our

687 results extend prior findings by introducing synonyms and homonyms and showing that

688 participants can learn these multiple mappings from the same implicit task but with no

689 differences between monolinguals and bilinguals. Since experimental work seldom targets other

690 aspects of language (e.g., grammar, syntax), it would be interesting to explore whether bilingual 
691 experience effects are constrained to the lexical level or extend to more higher-level aspects of a

692 foreign language.

\section{Conclusion}

694 Does bilingual experience influence statistical language learning? Our answer is that it

695 depends. Statistical learning is a robust cognitive process supported by a plethora of

696 experimental findings. At the same time, decisions about stimuli selection, experiment design,

697 participant selection, data collection, and statistical analysis can significantly influence the

698 outcome and interpretation of SL experiments. Our study explores only a handful of

699 manipulations that could have potentially elicited bilingual experience effects, such as

700 experience with different phonotactic patterns, the ability to generalize learned knowledge, and

701 the capacity to learn exclusive and multiple word-referent pairs. Crucially, SL only accounts for

702 a minimal part of what learning a new language entails. Adult individuals acquire a significant

703 part of a foreign language through explicit processes, and implicit learning might play a more

704 prominent role after individuals have achieved a certain proficiency level in a new language

705 (Ellis, 2015). However, the question of whether bilingual experience can influence SL as a

706 cognitive mechanism is perhaps ill-posed. Instead, to make progress in this field, we believe

707 studies should focus on how life experiences affect the strategies and filters through which

708 individuals implicitly process and learn from linguistic and non-linguistic material. 


\section{Acknowledgments}

711 This work has received funding from "la Caixa" Foundation and from the European Union's

712 Horizon 2020 research and innovation programme under the Marie-Skłodowska-Curie grant

713 agreement no. 713673, personal fellowship code LCF/BQ/IN17/116200154004, awarded to

714 JAA. MC was supported by the Basque Government through the BERC 2018-2021 program and

715 by the Spanish State Research Agency through BCBL Severo Ochoa excellence accreditation

716 SEV-2015-0490 and project RTI2018-093547-B-I00.

\section{Competing interests statement}

719 The authors declare no financial, commercial, or institutional competing interests.

\section{CRediT author contribution statement}

722 JAA: Conceptualization, Methodology, Software, Investigation, Resources, Data Curation, 723 Formal analysis, Writing - Original Draft, Visualization, Project administration, and Funding

724 acquisition MC: Conceptualization, Methodology, Writing - Review \& Editing, Supervision, 725 Project administration, and Funding acquisition.

\section{Open Practices statement}

728 Anonymized participant information, experimental data, materials, and annotated analyses

729 scripts for all experiments are freely available at https://osf.io/86sr4/. This study was not 730 preregistered. 


\section{References}

733 Alonso, R. A. (2016). Cross-linguistic influence in second language acquisition. In Multilingual Matters. https://doi.org/10.1002/9781405198431.wbeal0292

735 Antoniou, M., Liang, E., Ettlinger, M., \& Wong, P. C. M. (2015). The bilingual advantage in phonetic learning. Bilingualism, 18(4), 683. https://doi.org/10.1017/S1366728914000777

737 Assaneo, M. F., Ripollés, P., Orpella, J., Lin, W. M., de Diego-Balaguer, R., \& Poeppel, D. (2019). Spontaneous synchronization to speech reveals neural mechanisms facilitating language learning. Nature Neuroscience, 22(4), 627-632. https://doi.org/10.1038/s41593-

741 Barr, D. J., Levy, R., Scheepers, C., \& Tily, H. J. (2013). Random effects structure for confirmatory hypothesis testing: Keep it maximal. Journal of Memory and Language, 68(3),

744 Bartolotti, J., Marian, V., Schroeder, S. R., \& Shook, A. (2011). Bilingualism and inhibitory control influence statistical learning of novel word forms. Frontiers in Psychology, 2, 324. https://doi.org/10.3389/fpsyg.2011.00324

Bates, D., Mächler, M., Bolker, B. M., \& Walker, S. C. (2015). Fitting linear mixed-effects models using lme4. Journal of Statistical Software. https://doi.org/10.18637/jss.v067.i01

749 Bengtson, J. (2011). The Basque Language: History and Origin. International Journal of Modern Anthropology, 1(4), 43-59. https://doi.org/10.4314/ijma.v1i4.3

751 Benitez, V. L., Yurovsky, D., \& Smith, L. B. (2016). Competition between multiple words for a referent in cross-situational word learning. Journal of Memory and Language, 90, 31-48. https://doi.org/10.1016/j.jml.2016.03.004

754 de Bruin, A., Carreiras, M., \& Duñabeitia, J. A. (2017). The BEST dataset of language 
proficiency. Frontiers in Psychology, 8, 522. https://doi.org/10.3389/fpsyg.2017.00522

Donnelly, S., Brooks, P. J., \& Homer, B. D. (2015). Examining the Bilingual Advantage on Conflict Resolution Tasks: A Meta-Analysis. CogSci.

Dutoit, T., Pagel, V., Pierret, N., Bataille, E., \& van der Vrecken, O. (1996). MBROLA project: Towards a set of high quality speech synthesizers free of use for non commercial purposes. Proceeding of Fourth International Conference on Spoken Language Processing, ICSLP, 1393-1396. https://doi.org/10.1109/icslp.1996.607874

Ellis, N. C. (2015). Implicit AND explicit language learning. https://doi.org/10.1075/sibil.48.01ell

Endress, A. D., \& Bonatti, L. L. (2016). Words, rules, and mechanisms of language acquisition. Wiley Interdisciplinary Reviews: Cognitive Science, 7(1), 19-35. https://doi.org/10.1002/wcs.1376

Erickson, L. C., \& Thiessen, E. D. (2015). Statistical learning of language: Theory, validity, and predictions of a statistical learning account of language acquisition. Developmental Review, 37, 66-108. https://doi.org/10.1016/j.dr.2015.05.002

Escudero, P., Mulak, K. E., Fu, C. S. L., \& Singh, L. (2016). More limitations to monolingualism: Bilinguals outperform monolinguals in implicit word learning. Frontiers in Psychology, 7, 1218. https://doi.org/10.3389/fpsyg.2016.01218

Estes, K. G., Gluck, S. C. W., \& Grimm, K. J. (2016). Finding patterns and learning words: Infant phonotactic knowledge is associated with vocabulary size. Journal of Experimental Child Psychology, 146, 34-49. https://doi.org/10.1016/j.jecp.2016.01.012

Finn, A. S., \& Hudson Kam, C. L. (2008). The curse of knowledge: First language knowledge impairs adult learners' use of novel statistics for word segmentation. Cognition, 108(2), 
477-499. https://doi.org/10.1016/j.cognition.2008.04.002

779

780

781

782

783

784

785

786

787

788

789

790

791

792

793

794

795

796

797

798

799

800

Frost, R. L. A., \& Monaghan, P. (2016). Simultaneous segmentation and generalisation of nonadjacent dependencies from continuous speech. Cognition, 147, 70-74. https://doi.org/10.1016/j.cognition.2015.11.010

García Mayo, M. D. P., \& Villarreal Olaizola, I. (2011). The development of suppletive and affixal tense and agreement morphemes in the L3 english of basque-spanish bilinguals. Second Language Research, 27(1), 129-149. https://doi.org/10.1177/0267658310386523

Horst, J. S., \& Hout, M. C. (2016). The Novel Object and Unusual Name (NOUN) Database: A collection of novel images for use in experimental research. Behavior Research Methods, 48(4), 1393-1409. https://doi.org/10.3758/s13428-015-0647-3

Hurford, J. R. (2003). Why synonymy is rare: Fitness is in the speaker. Lecture Notes in Artificial Intelligence (Subseries of Lecture Notes in Computer Science), 442-451. https://doi.org/10.1007/978-3-540-39432-7_47

Jusczyk, P. W., Houston, D. M., \& Newsome, M. (1999). The Beginnings of Word Segmentation in English-Learning Infants. Cognitive Psychology, 39(3-4), 159-207. https://doi.org/10.1006/cogp.1999.0716

Kaufman, A. S., \& Kaufman, N. L. (2014). Kaufman Brief Intelligence Test, Second Edition. In Encyclopedia of Special Education. https://doi.org/10.1002/9781118660584.ese1325

Kaushanskaya, M., \& Marian, V. (2009). The bilingual advantage in novel word learning. Psychonomic Bulletin and Review, 16(4), 705-710. https://doi.org/10.3758/PBR.16.4.705

Kirkham, N. Z., Slemmer, J. A., \& Johnson, S. P. (2002). Visual statistical learning in infancy: Evidence for a domain general learning mechanism. Cognition, 83(2), 35-42. https://doi.org/10.1016/S0010-0277(02)00004-5 
801 Lemhöfer, K., \& Broersma, M. (2012). Introducing LexTALE: A quick and valid Lexical Test

802 for Advanced Learners of English. Behavior Research Methods, 44(2), 325-343. https://doi.org/10.3758/s13428-011-0146-0

Love, J., Selker, R., Marsman, M., Jamil, T., Dropmann, D., Verhagen, J., Ly, A., Gronau, Q. F., Šmíra, M., Epskamp, S., Matzke, D., Wild, A., Knight, P., Rouder, J. N., Morey, R. D., \& Wagenmakers, E. J. (2019). JASP: Graphical statistical software for common statistical

Maye, J., Werker, J. F., \& Gerken, L. A. (2002). Infant sensitivity to distributional information designs. Journal of Statistical Software, 88(1), 1-17. https://doi.org/10.18637/jss.v088.i02 can affect phonetic discrimination. Cognition, 82(3), 101-111.

McMurray, B., Aslin, R. N., \& Toscano, J. C. (2009). Statistical learning of phonetic categories: Insights from a computational approach. Developmental Science, 12(3), 369-378.

814 Mirman, D. (2017). Growth Curve Analysis and Visualization Using R. CRC press. https://doi.org/10.1201/9781315373218

Misyak, J., \& Christiansen, M. H. (2007). Extending statistical learning farther and further: Long-distance dependencies, and individual differences in statistical learning and language. Proceedings of the Annual Meeting of the Cognitive Science Society, 29(29).

Onnis, L., Chun, W. E., \& Lou-Magnuson, M. (2018). Improved statistical learning abilities in second-language reading efficiency. Proceedings of the 38th Annual Meeting of the Cognitive Science Society (CogSci 2016). 
824 Onnis, L., \& Thiessen, E. (2013). Language experience changes subsequent learning. Cognition, 126(2), 268-284. https://doi.org/10.1016/j.cognition.2012.10.008

Peirce, J., Gray, J. R., Simpson, S., MacAskill, M., Höchenberger, R., Sogo, H., Kastman, E., \& Lindeløv, J. K. (2019). PsychoPy2: Experiments in behavior made easy. Behavior Research Methods, 51(1), 195-203. https://doi.org/10.3758/s13428-018-01193-y

Peña, M., Bonatti, L. L., Nespor, M., \& Mehler, J. (2002). Signal-driven computations in speech processing. Science, 298(5593), 604-607. https://doi.org/10.1126/science.1072901

Perruchet, P., \& Pacton, S. (2006). Implicit learning and statistical learning: one phenomenon, two approaches. Trends in Cognitive Sciences, 10(5), 233-238. https://doi.org/10.1016/j.tics.2006.03.006

834 Pineño, O., \& Miller, R. R. (2005). Primacy and recency effects in extinction and latent inhibition: A selective review with implications for models of learning. Behavioural Processes, 69(2), 223-235. https://doi.org/10.1016/j.beproc.2005.02.006

Poepsel, T. J., \& Weiss, D. J. (2016). The influence of bilingualism on statistical word learning. Cognition, 152, 9-19. https://doi.org/10.1016/j.cognition.2016.03.001

Romberg, A. R., \& Saffran, J. R. (2010). Statistical learning and language acquisition. Wiley Interdisciplinary Reviews: Cognitive Science, 1(6), 906-914. https://doi.org/10.1002/wcs.78

841 Saffran, J. R. (2003). Statistical language learning: Mechanisms and constraints. Current Directions in Psychological Science, 12(4), 110-114. https://doi.org/10.1111/14678721.01243

844 Saffran, J. R., Aslin, R. N., \& Newport, E. L. (1996). Statistical learning by 8-month-old infants. Science, 274(5294), 1926-1928. https://doi.org/10.1126/science.274.5294.1926

846 Saffran, J. R., Johnson, E. K., Aslin, R. N., \& Newport, E. L. (1999). Statistical learning of tone 
sequences by human infants and adults. Cognition, 70(1), 27-52. https://doi.org/10.1016/S0010-0277(98)00075-4

Schad, D. J., Vasishth, S., Hohenstein, S., \& Kliegl, R. (2020). How to capitalize on a priori contrasts in linear (mixed) models: A tutorial. Journal of Memory and Language, 110, 104038. https://doi.org/10.1016/j.jml.2019.104038

\section{2}

853

Siegelman, N., Bogaerts, L., Christiansen, M. H., \& Frost, R. (2017). Towards a theory of individual differences in statistical learning. Philosophical Transactions of the Royal Society B: Biological Sciences, 372(1711), 20160059. https://doi.org/10.1098/rstb.2016.0059

Siegelman, N., \& Frost, R. (2015). Statistical learning as an individual ability: Theoretical perspectives and empirical evidence. Journal of Memory and Language, 81, 105-120. https://doi.org/10.1016/j.jml.2015.02.001

Smith, L., \& Yu, C. (2008). Infants rapidly learn word-referent mappings via cross-situational statistics. Cognition, 106(3), 1558-1568. https://doi.org/10.1016/j.cognition.2007.06.010

Thiessen, E. D., \& Saffran, J. R. (2003). When Cues Collide: Use of Stress and Statistical Cues to Word Boundaries by 7- to 9-Month-Old Infants. Developmental Psychology. https://doi.org/10.1037/0012-1649.39.4.706

van Doorn, J., van den Bergh, D., Böhm, U., Dablander, F., Derks, K., Draws, T., Etz, A., Evans, N. J., Gronau, Q. F., Haaf, J. M., Hinne, M., Kucharský, Š., Ly, A., Marsman, M., Matzke, D., Gupta, A. R. K. N., Sarafoglou, A., Stefan, A., Voelkel, J. G., \& Wagenmakers, E. J. (2020). The JASP guidelines for conducting and reporting a Bayesian analysis. Psychonomic Bulletin and Review, 1-14. https://doi.org/10.3758/s13423-020-01798-5 Wagenmakers, E. J. (2007). A practical solution to the pervasive problems of $\mathrm{p}$ values. 
871 Wang, T., \& Saffran, J. R. (2014). Statistical learning of a tonal language: The influence of

872 bilingualism and previous linguistic experience. Frontiers in Psychology, 5, 953.

873 https://doi.org/10.3389/fpsyg.2014.00953

874 Yu, C., \& Smith, L. B. (2007). Rapid word learning under uncertainty via cross-situational

$875 \quad$ statistics. Psychological Science, 18(5), 414-420. https://doi.org/10.1111/j.1467-

$876 \quad 9280.2007 .01915 . x$

877 
Appendix A

879 Linguistic profile and contrasts per group in Experiment 1

\begin{tabular}{|c|c|c|c|c|c|c|c|c|c|c|c|}
\hline & \multicolumn{2}{|c|}{$\begin{array}{c}\text { Spanish } \\
\text { Monolinguals } \\
\end{array}$} & \multicolumn{2}{|c|}{$\begin{array}{c}\text { Spanish-Basque } \\
\text { Bilinguals }\end{array}$} & \multicolumn{2}{|c|}{$\begin{array}{c}\text { Spanish-English } \\
\text { Bilinguals }\end{array}$} & \multicolumn{3}{|c|}{ ANOVA } & \multicolumn{2}{|c|}{ Helmert contrasts $\mathrm{p}$-value } \\
\hline & $M$ & $S D$ & $M$ & $S D$ & $M$ & $S D$ & $\mathrm{~F}_{(2,114)}$ & $p$ & $\mathrm{BF}_{10}$ & MONO-BIL & SPBQ-SPEN \\
\hline Age & 21.8 & 2.6 & 21.2 & 1.9 & 20.9 & 2.3 & 1.610 & 0.204 & 0.303 & 0.088 & 0.586 \\
\hline Non-verbal IQ & 101.5 & 8.3 & 100.7 & 8.6 & 99.8 & 6.6 & 0.481 & 0.619 & 0.120 & 0.406 & 0.592 \\
\hline Age of Acquisition L1 ${ }^{a}$ & 0.0 & 0.0 & 0.0 & 0.0 & 0.0 & 0.0 & - & - & - & - & - \\
\hline Total Exposure L1 (\%) & 91.4 & 8.1 & 60.3 & 11.8 & 64.5 & 9.9 & 111.685 & $<0.001$ & $>100$ & $<0.001$ & 0.070 \\
\hline Self-rated proficiency L1 (1-10) & 9.8 & 0.4 & 9.8 & 0.2 & 9.9 & 0.3 & 0.876 & 0.419 & 0.166 & 0.411 & 0.294 \\
\hline BEST L1 (0-65) & 64.6 & 0.6 & 64.6 & 0.7 & 64.8 & 0.5 & 1.425 & 0.245 & 0.260 & 0.217 & 0.243 \\
\hline Age of Acquisition L2 & 5.3 & 1.8 & 3.0 & 1.6 & 3.5 & 1.7 & 20.508 & $<0.001$ & $>100$ & $<0.001$ & 0.237 \\
\hline Total Exposure L2 (\%) & 7.7 & 7.2 & 32.5 & 13.1 & 33.5 & 9.4 & 80.766 & $<0.001$ & $>100$ & $<0.001$ & 0.664 \\
\hline Self-rated proficiency L2 (1-10) & 4.3 & 1.8 & 8.6 & 0.7 & 8.1 & 0.8 & 149.854 & $<0.001$ & $>100$ & $<0.001$ & 0.074 \\
\hline BEST L2 (0-65) & 25.9 & 8.2 & 55.9 & 6.9 & 55.9 & 4.2 & 264.291 & $<0.001$ & $>100$ & $<0.001$ & 0.991 \\
\hline LexTALE L1 (\%) & - & - & 92.9 & 5.6 & 93.8 & 6.3 & - & - & - & - & $0.506^{\mathrm{b}}$ \\
\hline LexTALE L2 (\%) & - & - & 90.7 & 6.1 & 88.8 & 6.0 & - & - & - & - & $0.176^{\mathrm{b}}$ \\
\hline
\end{tabular}

880 Note. Significant contrast terms are highlighted in bold. SD = standard deviation; MONO = Spanish monolinguals; BIL = bilinguals; SPEN = Spanish-English

881 bilinguals; SPBQ = Spanish-Basque bilinguals.

$882 \quad{ }^{a}$ Statistic and $p$-value undefined due to zero variance.

$883{ }^{\mathrm{b}}$ Difference calculated using Welch t-tests. 
886 Linguistic profile and contrasts per group in Experiment 2



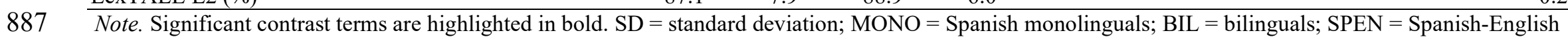

888 bilinguals; SPBQ = Spanish-Basque bilinguals.

889 a Statistic and $p$-value undefined due to zero variance.

890 b Difference calculated using Welch t-tests. 
894 Linguistic profile and contrasts per group in Experiment 3

\begin{tabular}{|c|c|c|c|c|c|c|c|c|c|c|c|}
\hline & \multicolumn{2}{|c|}{$\begin{array}{c}\text { Spanish } \\
\text { Monolinguals } \\
(\mathrm{n}=40)\end{array}$} & \multicolumn{2}{|c|}{$\begin{array}{c}\text { Spanish-Basque } \\
\text { Bilinguals } \\
(\mathrm{n}=40)\end{array}$} & \multicolumn{2}{|c|}{$\begin{array}{c}\text { Spanish-English } \\
\text { Bilinguals } \\
(\mathrm{n}=37) \\
\end{array}$} & \multicolumn{3}{|c|}{ ANOVA } & \multicolumn{2}{|c|}{ Helmert contrasts p-value } \\
\hline & $M$ & $S D$ & $M$ & $S D$ & $M$ & $S D$ & $F_{(2,114)}$ & $p$ & $\mathrm{BF}_{10}$ & MONO-BIL & SPBQ-SPEN \\
\hline Age & 21.7 & 2.5 & 21.9 & 1.9 & 21.0 & 2.4 & 1.712 & 0.185 & 0.328 & 0.696 & 0.072 \\
\hline Non-verbal IQ & 102.2 & 8.0 & 101.5 & 6.2 & 100.6 & 6.5 & 0.477 & 0.622 & 0.120 & 0.313 & 0.583 \\
\hline Age of Acquisition L1 ${ }^{a}$ & 0.0 & 0.0 & 0.0 & 0.0 & 0.0 & 0.0 & - & - & - & - & - \\
\hline Total Exposure L1 (\%) & 90.7 & 7.5 & 62.5 & 10.8 & 65.0 & 9.4 & 111.191 & $<0.001$ & $>100$ & $<0.001$ & 0.242 \\
\hline Self-rated proficiency L1 (1-10) & 9.9 & 0.3 & 9.8 & 0.3 & 9.9 & 0.2 & 1.54 & 0.219 & 0.286 & 0.962 & 0.082 \\
\hline BEST L1 (0-65) & 64.7 & 0.5 & 64.7 & 0.5 & 64.8 & 0.4 & 1.618 & 0.203 & 0.304 & 0.145 & 0.284 \\
\hline Age of Acquisition L2 & 5.5 & 2.0 & 2.9 & 1.0 & 3.6 & 1.7 & 26.798 & $<0.001$ & $>100$ & $<0.001$ & 0.067 \\
\hline Total Exposure L2 (\%) & 8.9 & 7.2 & 30.5 & 9.3 & 33.1 & 8.9 & 95.487 & $<0.001$ & $>100$ & $<0.001$ & 0.183 \\
\hline Self-rated proficiency L2 (1-10) & 3.9 & 1.6 & 8.5 & 0.9 & 8.1 & 0.9 & 180.184 & $<0.001$ & $>100$ & $<0.001$ & 0.082 \\
\hline BEST L2 (0-65) & 26.9 & 9.2 & 56.2 & 7.3 & 55.7 & 4.3 & 210.094 & $<0.001$ & $>100$ & $<0.001$ & 0.766 \\
\hline LexTALE L1 (\%) & - & - & 91.1 & 7.6 & 93.9 & 5.9 & - & - & - & - & $0.077^{\mathrm{b}}$ \\
\hline LexTALE L2 (\%) & - & - & 88.7 & 8.9 & 88.9 & 6.1 & - & - & - & - & $0.900^{\mathrm{b}}$ \\
\hline
\end{tabular}

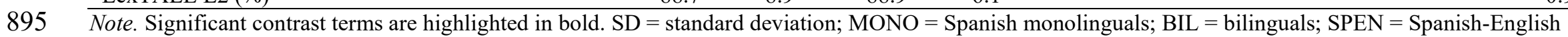

896 bilinguals; SPBQ = Spanish-Basque bilinguals.

897 a Statistic and $p$-value undefined due to zero variance.

$898 \quad$ b Difference calculated using Welch t-tests. 
